\title{
Carcinógenos ambientales asociados a cáncer infantil
}

\author{
Environmental carcinogens associated with childhood cancer \\ María Sofía González-Casagua' orcid.org/0000-0002-4023-3517 \\ Juan David Bermeo-Calderón' orcid.org/0000-0002-0066-5761 \\ Luis Alfonso Laverde-Gaona' ${ }^{1}$ orcid.org/0000-0003-1 162-4524 \\ Yaliana Tafurt-Cardona ${ }^{*}$ orcid.org/0000-0003-1289-2395
}

1 Grupo de Investigación Navarra Medicina, Facultad de Ciencias de la Salud, Fundación Universitaria Navarra - UNINAVARRA. Neiva, Colombia.

\begin{abstract}
Resumen
Introducción: La Sociedad Americana de Cáncer indica que al año se diagnostican 163.300 casos de cáncer infantil en el mundo. En Colombia fueron reportadas 300 muertes por esta causa en menores de edad durante el año 2015. Actualmente, las principales asociaciones etiológicas de cáncer infantil son la radiación ionizante y exposición a pesticidas, convirtiéndose en una prioridad emergente en la agenda mundial de salud infantil. Objetivo: Identificar factores carcinogénicos asociados al incremento de riesgo en la aparición de cáncer infantil. Materiales y métodos: Se realizó una revisión de artículos científicos en inglés y español en la base de datos PubMed, ScienceDirect, SciELO, y publicaciones estadísticas de la Organización Mundial de la Salud, Asociación Americana de Cáncer y el Instituto Nacional de Cancerología de Colombia. Resultados: Se describieron diferentes factores carcinogénicos como radiación ionizante, agentes biológicos, patrones dietéticos, exposición a pesticidas, tabaco y asbesto, destacando su asociación en el desarrollo de cáncer infantil. Conclusión: El reconocimiento de los agentes carcinogénicos frecuentemente asociados con cáncer infantil, permite identificar el impacto de estos sobre la salud, y generar medidas preventivas más eficaces que puedan reducir la carga global de la enfermedad.
\end{abstract}

Palabras clave: Niño; neoplasias; carcinógenos; factores de riesgo; exposición a riesgos ambientales. (Fuente: DeCS, Bireme).

\begin{abstract}
Introduction: The American Cancer Society indicates that each year 163,300 cases of childhood cancer are diagnosed worldwide. In Colombia, 300 deaths were reported from this cause in minors during 2015. Currently, the main etiological associations of childhood cancer are ionizing radiation and exposure to pesticides, making it an emerging priority in the global agenda for children's health. Objective: To identify carcinogenic factors associated with the increased risk in the onset of childhood cancer. Materials and methods: A review of scientific articles in English and Spanish was carried out in PubMed, ScienceDirect, SciELO, and statistical publications of the World Health Organization, American Cancer Association and the National Cancer Institute of Colombia. Results: Different carcinogenic factors were described as ionizing radiation, biological agents, dietary patterns, exposure to pesticides, tobacco and asbestos, highlighting their association in the development of childhood cancer. Conclusion: The recognition of the carcinogenic agents frequently associated with childhood cancer, allows the identification of their impact on health, and generates more effective preventive measures that can reduce the worldwide burden of the disease.
\end{abstract}

Key words: Child; neoplasms; carcinogens; risk factors; environmental exposure. (Source: DeCS, Bireme). 


\section{Introducción}

Desde 1946 la Organización Mundial de la Salud (OMS), destacó el riesgo asociado al desequilibrio entre factores genéticos y ambientales en el desarrollo de enfermedades no trasmisibles con una carga global de enfermedades del $31 \%$ a $41 \%(1)$, afectando principalmente a la población infantil menor de cinco años, con aproximadamente 223 millones de defunciones en los últimos 20 años(2), convirtiéndose en una prioridad emergente de la agenda mundial en salud infantil(1).

Se estima que anualmente se diagnostican 163.300 casos de cáncer infantil en el mundo y en promedio uno de cada 408 niños menores de 15 años y uno de cada 285 niños entre los 15 y 20 años terminan en decesos, es decir 80.000 defunciones al año(3). En América Latina y el Caribe, 17.500 nuevos casos son diagnosticados cada año y más de 8.000 muertes se registran por esta enfermedad(4).

En Colombia, fueron reportadas 300 muertes por esta causa en menores de edad durante 2015(5). Los registros poblacionales de cáncer y los datos de mortalidad de la Secretaría de Salud Pública de Cali, registraron 2.311 casos entre 1977 y 2011, de los cuales el 54,5\% fueron en hombres y el $40,2 \%$ en menores de cinco años ${ }^{(4)}$.

Según la OMS la morbilidad asociada a enfermedades no transmisibles en la infancia está en incremento(1), lo cual puede estar vinculado a la interacción de factores bio-físico-químicos, psicosociales, culturales y económicos(6). La etiología del cáncer infantil está asociada principalmente a la radiación ionizante y a las diversas alteraciones genéticas hereditarias que representan entre el $5 \%$ y el $10 \%$ de los casos identificados(7).

Los factores ambientales desempeñan un importante papel en el desarrollo de las diferentes neoplasias, principalmente en los primeros años de vida, debido a la vulnerabilidad producida por la biotransformación del hígado para metabolizar eficientemente las toxinas, las altas tasas de absorción de productos químicos, los mecanismos de reparación del ADN y las grandes tasas de proliferación celular; sin embargo, existe la necesidad de identificar los factores de riesgo no genéticos en este grupo etario e implementar el desarrollo de medidas preventivas específicas(7). Se ha demostrado el potencial carcinogénico postexposición a factores exógenos específicos relacionados con los casos de cáncer infantil(3,7).

El presente artículo, tiene por objetivo identificar factores carcinogénicos asociados al incremento del riesgo en la aparición de cáncer infantil.

\section{Materiales y métodos}

Se realizó una revisión de tema por medio de la búsqueda de literatura disponible en PubMed, ScienceDirect, SciELO y publicaciones estadísticas de la OMS, la Asociación Americana de Cáncer y el Instituto Nacional de Cancerología de Colombia. Los criterios de inclusión que se tuvieron en cuenta para la búsqueda y selección fueron: artículos de investigación finalizados y publicados en las bases de datos mencionadas anteriormente, entre los años 2013 a 2018 en idioma español e inglés usando las palabras clave en español: Niño, neoplasias, carcinógenos, factores de riesgo, exposición a riesgos ambientales y las palabras en inglés: Child, neoplasms, carcinogens, risk factors, environmental exposure, que se combinaron para obtener resultados acordes al objetivo de la investigación. Finalmente, se seleccionaron 56 artículos que cumplieron con los criterios de inclusión, se realizó la descripción de factores carcinogénicos como: radiación ionizante, patrón dietético, pesticidas, asbesto, tabaco y agentes biológicos.

\section{Resultados}

\section{Radiación ionizante}

Es considerada un agente carcinogénico dosis dependiente, capaz de transferir energía altamente estructurada de eventos de ionización y excitación que pueden producir daños complejos al ADN, tales como lesión en bases simples, alteraciones monocatenarias en el esqueleto del ADN, rotura del ADN bicatenario, aberraciones cromosómicas, cambios celulares, mutaciones y muerte celular(8-10). La Agencia Internacional para la Investigación del Cáncer (IARC), clasifica la radiación ionizante como carcinogénico del grupo 1 , con alto riesgo de producir neoplasias que comprometen principalmente el tegumento, tiroides, cerebro, mamas y sangre, no sólo por la exposición ambiental sino por el efecto iatrogénico por el uso 
en métodos diagnósticos $\mathrm{y}$ tratamiento contra neoplasias(11-13).

En Estados Unidos se demostró mediante la evaluación de un grupo de 1255 menores de 20 años, una relación directamente proporcional entre el aumento de la radiación UV y el incremento del riesgo a desarrollar melanoma, debido a la transición de citidina a timidina, que genera dímeros de pirimidina de ciclobutano en el ADN(14). En niños con exposición previa a radiación ionizante se ha observado que los tumores malignos secundarios más comunes fueron cáncer de piel no melanoma, cáncer de mama y tiroides, tumores óseos y tumores benignos del SNC, como meningiomas.(15). Una reciente investigación identifica una relación lineal dosis-respuesta entre la exposición a yodo reactivo y el cáncer de tiroides ${ }^{(16)}$

Investigaciones en la población infantil tras el desastre nuclear de Chernobyl concluyen que las consecuencias por sobreexposición a la radiación ionizante incluyen trastornos inmunológicos, enfermedades cardiovasculares y aumento del riesgo a neoplasias, principalmente leucemias, cáncer de mama, tiroides, piel y cerebro, debido a una mayor tasa de división celular, tejidos más blandos, órganos más contiguos, mayor susceptibilidad a factores ambientales y menor diámetro corporal(17-20).

La IARC clasifica los campos electromagnéticos de radiofrecuencia como posibles carcinogénicos para los seres humanos, con un riesgo asociado a la exposición de campos magnéticos superiores a 0,4 $m$ que duplica el riesgo de desarrollar leucemia; sin embargo, no hay evidencia suficiente de asociación entre la radiación no ionizante de baja frecuencia y un mayor riesgo de desarrollar tumores cerebrales en la población infantil y adolescente expuesta a campos electromagnéticos de radiofrecuencia de los teléfonos móviles(21).

\section{Patrón dietético}

La exposición a mutágenos dietéticos y mutagénicos por deficiencia de nutrientes aumenta el riesgo de desarrollar procesos neoplásicos en la infancia. La asociación entre las tasas de cáncer y los patrones dietéticos varía significativamente entre la población de diferentes países: aproximadamente el 30 \% de los cánceres en los países occidentales está asociado a la alta ingesta de productos de origen animal, grasas y azúcares; en contraste, probablemente por el déficit dietético de la población de países en vía de desarrollo, se reportan menos del $20 \%$ del total de casos en el mundo y se relacionan, además, con la ingesta de alimentos contaminados como la micotoxina aflatoxina(22).

El déficit de nutrientes como riboflavina, ácido fólico, ácido ascórbico y zinc, la elevada ingesta de carnes rojas procesadas y la alimentación formulada 14 días después del nacimiento se asocian con el riesgo a desarrollar leucemia infantil; mientras que el consumo de alimentos ricos en sal incrementa el riesgo de padecer cáncer colorrectal y de estómago(23,24).

Se han identificado factores protectores relacionados con el patrón dietético y la reducción del riesgo de cáncer, tales como la lactancia materna por ingesta de nutrientes esenciales para el recién nacido y cebados inmunológicos, que reducen el riesgo de desarrollar leucemias en los niños(25); una dieta abundante en verduras $y$ frutas aporta vitaminas y minerales anti-cancerosos, antiproliferativos, y anti-inflamatorios, que reducen el riesgo de cáncer de esófago, estómago y cáncer colorrectal $(23,24,26)$; el consumo regular de naranjas y bananos durante los dos primeros años de vida reduce el riesgo de desarrollar leucemia infantil(26).

Se relacionan factores dietéticos maternos en la etapa pregestacional y prenatal con el riesgo a desarrollar leucemia linfoblástica aguda en la infancia(27). Estudios en gemelos monocigóticos que desarrollaron leucemia se destacaron dos etapas específicas relacionadas con la aparición de cáncer, en primer lugar, un proceso de translocación cromosómicas prenatal, etapa preleucémica, y una exposición postnatal a factores extrínsecos genotóxicos esenciales para desarrollar cambios genéticos secundarios compatibles con leucemia(28).

La dieta materna constituida por la ingesta de vegetales, frutas, alimentos con alto contenido de carotenoides (zanahoria y melón), antioxidante glutatión (carne de res y los frijoles), pescado y mariscos reducen el riesgo de leucemia linfoblástica aguda(29), además, la ingesta materna de ácido fólico $\mathrm{y}$ vitaminas $\mathrm{B}$-cofactores determinantes del metabolismo del carbono- influyen en los procesos de síntesis, reparación genética del ADN e integridad cromosómica que establece una relación 
inversa con el riesgo de desarrollar leucemia infantil(30,31).

\section{Pesticidas}

La IARC clasifica los plaguicidas como posibles carcinogénicos en humanos, produciendo estrés oxidativo, aberraciones cromosómicas, genotoxicidad, alteraciones endocrinas, inhibición de la colinesterasa y mutaciones de señalización en células embrionarias o somáticas, con efectos principalmente en niños y relacionados con la rápida división celular de sus tejidos, las actividades al aire libre, una alta ingesta de alimentos o líquidos por libra de peso corporal y la incompleta función enzimática desintoxicante(32-36). Se ha descrito que incrementan el riesgo de leucemia infantil, cáncer cerebral, tumor de Wilms, sarcoma de Ewing y tumores de células germinales(32,37).

Los niños que habitan en zonas de cultivo, están expuestos a plaguicidas por lo tanto tienen mayor riesgo de desarrollar neoplasias, debido a la dispersión de partículas de polvo de estos químicos que contaminan el exterior e interior de los hogares(32). Los insecticidas de uso doméstico como repelentes, productos de higiene animal y champús antipiojos pueden depositarse en juguetes infantiles, facilitando así la contaminación y aumento de riesgo a desarrollar leucemia infantil(32,33). Otras vías de contaminación menos frecuentes se relacionan con el consumo de alimentos y líquidos con altos residuos de plaguicidas; sin embargo, la concentración de partículas no sobrepasa las altas concentraciones encontradas en el polvo doméstico(34).

El Consorcio Internacional de Leucemia Infantil (CLIC, por sus siglas en inglés) evidenció una asociación por exposición ocupacional preconcepcional de los padres a pesticidas con el aumento del riesgo a desarrollar leucemias infantiles, la exposición paterna se relacionó con el desarrollo de leucemia linfoblástica aguda, mientras que la exposición materna se asoció con leucemia mieloblástica aguda, con una prevalencia de 30\% y $90 \%$ respectivamente ${ }^{(38-40)}$.

\section{Asbesto}

La Sociedad Americana contra el cáncer declararon que el asbesto es un carcinogénico humano(41). En España, el principal agente desencadenante de cáncer pulmonar es el asbesto, determinándose que el tiempo de latencia entre la exposición del agente y la detección clínica de la enfermedad es cerca de 40 años; aunque, en la mayoría de las enfermedades carcinogénicas el intervalo es alrededor de 12 a 15 años ${ }^{(42-44) .}$

Según cifras de comercialización de asbesto publicada por el Servicio Geológico de los Estados Unidos, en Colombia aumentó un $26 \%$ el consumo de este material para el año 2012 y en la actualidad este material se sigue utilizando en la industria y construcción(45). Como evidencia están muchas de las viviendas de interés social o comunitario, en las cuales algunos de sus materiales contienen asbesto, ignorando o menospreciando que es un potencial factor de riesgo carcinogénico para la población infantil(46).

El asbesto ocasiona daños al ADN por la acción de radicales hidroxilos y el bloqueo de la citocinesis, provocando cambios en el genoma, los cuales desencadenan una transformación neoplásica y progresión de células tumorales, causando cáncer pulmonar, mesotelioma pleural y asbestosis $(47,48)$. Se ha documentado una relación directamente proporcional entre la exposición de asbesto a temprana edad y elevadas tasas de mortalidad por mesotelioma y también relacionan la duración y la dosis acumulada de exposición al asbesto con el riesgo de cáncer pulmonar(49,50).

La exposición al asbesto durante la niñez se debe a la proximidad de las industrias y a su uso en la construcción de casas, principalmente en escuelas y parques escolares, pavimento de carreteras y zonas de estacionamiento, especialmente a países en vía de desarrollo(51). Adicionalmente, otra forma de exposición en niños menores de cinco años es comer tierra o polvo contaminados con asbesto, inhalación de fibras de asbesto a causa de familiares expuestos laboralmente, trabajando en minas o industrias. Sin embargo, las investigaciones respecto a la asociación del cáncer en la infancia y el asbesto son inconclusas y limitadas(51).

\section{Tabaco}

Cerca de 1000 millones de hombres y 250 millones de mujeres fuman en todo el planeta, aproximadamente entre 82000 y 99000 jóvenes, son nuevos fumadores cada año, muchos de ellos menores de 10 años(52,53). La Comunidad Europea plantea que el tabaquismo pasivo es el principal 
factor de riesgo de enfermedades respiratorias crónica y carcinogénicas(54). La Encuesta mundial sobre el tabaco reporta que casi la mitad de los niños que nunca habían fumado están expuestos al tabaquismo pasivo, tanto en su casa como fuera de ella(55).

La IARC asocia la exposición al tabaco como factor desencadenante de neoplasias en la población infantil, así como de infecciones respiratorias, disfunciones pulmonares y asma, que se favorecen por la inmadurez anatomo-fisiológica de sus

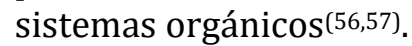

El tabaco es un potencial carcinogénico con componentes tóxicos como el benceno, formaldehído, hidrocarburos aromáticos policíclicos y el polonio, causando daños epiteliales, inflamación e irritación(58,59). El benceno se asocia a daños en la línea mieloide y linfoide de las células hematopoyéticas, aumentando el riesgo de desarrollar leucemia linfoide o mieloide

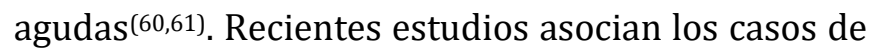
leucemia linfoblástica aguda en menores de 15 años con la exposición al tabaco, derivada de la compra ilegal de este producto, pese a las disposiciones legales que prohíben su venta a menores de 18 años (62-64).

\section{Agentes biológicos}

La exposición a agentes biológicos infecciosos en la infancia se asocia con un mayor riesgo de malignidad a través del curso de la vida, relacionado a la inmadurez fisiológica y la vulnerabilidad en las etapas críticas del desarrollo(65). La IARC identificó agentes biológicos potencialmente carcinogénicos en humanos con características y comportamiento específicos dependiendo de las condiciones socioeconómicas que faciliten la infección durante la infancia(33).

La infección por virus Epstein-Barr (EBV) es universal y se relaciona con el riesgo a desarrollar leucemia infantil, específicamente, leucemia linfoblástica aguda, linfoma de Burkitt y linfoma de Hodgkin infantil en un 90\%; en los países en vía de desarrollo afecta principalmente a la población infantil en contraposición con los países desarrollados que reportan mayores casos de infección en la población adolescente ${ }^{(66,67)}$.
Por otra parte, se estima que el $30 \%$ de la población infantil está infectada por Helicobacter pylori, principalmente entre los 3 y 5 años, la infección se asocia con ulceraciones en la mucosa gástrica que aumentan el riesgo para el desarrollo de linfoma gástrico tipo Linfoma $\mathrm{T}$, con una mayor presentación clínica en la vida adulta; la infección en la infancia se asocia al hacinamiento, condiciones sanitarias deficientes, ausencia de agua potable y consumo de alimentos contaminados(68,69).

\section{Conclusiones}

Este artículo recopiló aspectos relevantes sobre carcinogénicos ambientales asociados a morbimortalidad por cáncer infantil, especialmente por alta ingesta de productos de origen animal, grasas y azúcares, la exposición a radiación ionizante, a pesticidas, a productos de higiene animal y la presencia de asbesto en pavimento de carreteras, zonas de estacionamiento de escuelas y parques escolares. La evidencia científica demuestra el desconocimiento del riesgo atribuido a cada factor y sus elementos, que pueden ocasionar aberraciones cromosómicas, genotoxicidad, cambios celulares, mutaciones y muerte celular; con repercusiones en la calidad de vida a corto y largo plazo de los niños afectados y su impacto en el estado socioeconómico de las familias involucradas.

El reconocimiento de los agentes carcinogénicos permite identificar el impacto de estos sobre la salud, por lo cual, es imprescindible conocer los diferentes factores de riesgo de la población infantil $\mathrm{y}$ de esta forma generar medidas preventivas y de control más eficaces y específicas que puedan reducir la carga global de la enfermedad.

\section{Conflicto de intereses}

Los autores no declaran conflictos de interés.

\section{Referencias}

1. Organización Mundial de la Salud. Reducción de la mortalidad en la niñez. Ginebra: OMS; 2018 [Internet]. Disponible http://www.who.int/mediacentre/factsheets/fs178/es/

2. United Nations Children's Fund. Compromisso com a sobrevivência infantil: uma promessa. Relatório de progresso 2014. Resumo executivo. Brasil: Unicef; 2014. [Internet]. [citado 16 de noviembre de 2017]. Disponible en: http://www.unicef.pt/promessa-renovada2014/unicef-relatorio-promessa-renovada-2014.html 
3. American Cancer Society. Global Cancer Facts \& Figures [Internet] 3rd Edition; USA: ACS; 2015. [citado 22 de enero del 2018]. Disponible en: https://www.cancer.org/content/dam/cancer$\mathrm{org} /$ research/cancer-facts-and-statistics/global-cancerfacts-and-figures/global-cancer-facts-and-figures-3rdedition.pdf

4. Bravo LE, García LS, Collazos P, Aristizabal P, Ramirez O. Descriptive epidemiology of childhood cancer in Cali. Colomb Médica CM. 2013; 44(3):155-64.

5. Instituto Nacional de Cancerología ESE. Análisis de la Situación del Cáncer en Colombia 2015. Primera edición. Bogotá DC: Instituto Nacional de Cancerología ESE; 2017. [Internet]. [citado 23 de abril del 2018]. Disponible en: http://www.cancer.gov.co/Situacion_del_Cancer_en_Colom bia_2015.pdf

6. Organización Mundial de la Salud. 10 datos sobre los niños y la higiene del entorno. [Internet]. Ginebra: OMS; 2017 [citado 10 de octubre del 2017]. Disponible en: http://www.who.int/features/factfiles/children_environm ental_health/es/

7. Norman RE, Sample Organization. Environmental Contributions to Childhood Cancers. I Enviromental Immunol Toxicol. 2014;2(2):86-98.

8. González-Meneses A. Bases genéticas y moleculares en el cáncer infantil, Pediatr Integral 2016;XX(6):359-366.

9. Leuraud, K. et al. Ionising radiation and risk of death from leukaemia and lymphoma in radiation-monitored workers (INWORKS): an international cohort study. Lancet Haematol. 2015;2:e276-e281.

10. Ravanat, JL, Breton J, Douki T, Gasparutto D, Grand A, Rachidi W, et al. Radiation-mediated formation of complex damage to DNA: a chemical aspect overview. Br. J. Radiol. 2014; 87(1035):20130715. doi: 10.1259/bjr.20130715.

11. Rubin, G.D. Computed tomography: revolutionizing the practice of medicine for 40 years. Radiology. 2014;273:S45-S47.

12. Pandharipande PV, Reisner AT, Binder WD, Zaheer A, Gunn ML, Linnau KF, et al. CT in the emergency department: a real-time study of changes in physician decision making. Radiology 2016;278:812-821.

13. Pandharipande PV, Alabre CL, Coy DL, Zaheer A, Miller CM, Herring MS, et al. Changes in physician decision making after CT: a prospective multicenter study in primary care settings. Radiology. 2016;281:835-846.

14. Gefeller 0 , Uter W, Pfahlberg AB. Protection from Ultraviolet Radiation during Childhood: The Parental Perspective in Bavaria. Int J Environ Res Public Health. 2016;13(10):1011.

15. Whelan K, Alva E. Pediatric Cancer Genetics. Chapter 1, Epidemiology of Childhood Cancer. Elsevier; 2018. p. 1-20

16. Yamashita S, Takamura N, Ohtsuru A, Suzuki S. Radiation Exposure and Thyroid Cancer Risk After the Fukushima Nuclear Power Plant Accident in Comparison with the Chernobyl Accident. Radiat Prot Dosimetry. 2016;171(1):41-6.

17. Bakhmutsky MV, Joiner MC, Jones TB, Tucker JD. Differences in cytogenetic sensitivity to ionizing radiation in newborns and adults. Radiat Res 2016; 181(6):605-616.

18. Fucic A, Aghajanyan A, Druzhinin V, Minina V, Neronova E. Follow-up studies on genome damage in children after Chernobyl nuclear power plant accident. Arch Toxicol. 2016;90(9):2147-2159.
19. Zablotska LB, Nadyrov EA, Polyanskaya ON, McConnell RJ, O'Kane P, Lubin J, et al. Risk of thyroid follicular adenoma among children and adolescents in Belarus exposed to iodine-131 after the Chornobyl accident. Am J Epidemiol 2015;182(9):781-790.

20. Ariyoshi K, Miura T, Kasai K, Akifumi N, Fujishima Y, Yoshida MA. Age Dependence of Radiation-Induced Genomic Instability in Mouse Hematopoietic Stem Cells. Radiat Res. 2018. doi: 10.1667/RR15113.1

21. Miah T, Kamat D. Current Understanding of the Health Effects of Electromagnetic Fields. Pediatr Ann. 2017;1;46(4):e172-e174.

22. Clarke MA, Joshu CE. Early Life Exposures and Adult Cancer Risk. Epidemiol Rev. 2017;39(1):11-27.

23. Greenop KR, Bailey HD, Miller M Breastfeeding and nutrition to 2 years of age and risk of childhood acute lymphoblastic leukemia and brain tumors. Nutr Cancer. 2015;67(3):431-41.

24. Moulik NR, Kumar A, Agrawal S. Folic acid, one-carbon metabolism \& childhood cancer. Indian J Med Res. 2017;146(2):163-174.

25. Whitehead T, Metayer C, Wiemels J, Singer A, Miller M, Childhood Leukemia and Primary Prevention. Curr Probl Pediatr Adolesc Health Care. 2016; 46:317-352.

26. Nimptsch K, Malik VS, Fung TT, et al. Dietary patterns during high school and risk of colorectal adenoma in a cohort of middle-aged women. Int $\mathrm{J}$ Cancer. 2014;134(10):2458-2467.

27. Singer AW, Carmichael SL, Selvin S, Fu C, Block G, Metayer C. Maternal diet quality before pregnancy and risk of childhood leukaemia. Br J Nutr. 2016;116(8):1469-1478.

28. Swaminathan S, Klemm L, Park E, Papaemmanuil E, Ford A, Kweon SM, et al. Mechanisms of clonal evolution in childhood acute lymphoblastic leukemia. Nat Immunol. 2015;16(7):766-774.

29. Abiri B, Kelishadi R, Sadeghi H, Azizi-Soleiman F. Effects of Maternal Diet During Pregnancy on the Risk of Childhood Acute Lymphoblastic Leukemia: A Systematic Review. Nutr Cancer. 2016;68(7):1065-72.

30. Locasale JW. Serine, glycine and one-carbon units: cancer metabolism in full circle. Nature reviews. Cancer. 2013;13(8):572-583.

31. Singer AW, Selvin S, Bloque G, C Dorado, Carmichael SL, Metayer C. Maternal prenatal intake of one-carbon metabolism nutrients and risk of childhood leukemia. Cancer Causes Control. 2016;27(7):929-40.

32. Greenop KR, Peters S, Bailey HD, Fritschi L, Attia J, Scott RJ, et al. Exposure to pesticides and the risk of childhood brain tumors. Cancer Causes Control. 2013;24(7):1269-78.

33. International Agency for Research on Cancer (IARC). Monographs on the Evaluation of Carcinogenic Risks to Humans. Lyon: IARC; 2016. [Internet]. [citado 10 de Enero del 2018]. Disponible en: http://monographs.iarc.fr/.

34. Oulhote Y, Bouchard MF. Urinary metabolites of organophosphate and pyrethroid pesticides and behavioral problems in Canadian children. Environ Health Perspect. 2013;121:1378-1384

35. Choi J, Polcher A, Joas A. Systematic literature review on Parkinson's disease and Childhood Leukaemia and mode of actions for pesticides. European Food Safety Authority Supporting Pubblications. 2016;En-955:1-4.

36. Hernandez AF, Menendez P. Linking Pesticide Exposure with Pediatric Leukemia: Potential Underlying Mechanisms. Int J Mol Sci. 2016; 29;17(4):461. 
37. Chen $\mathrm{M}$, Chang $\mathrm{CH}$, Tao $\mathrm{L}$, et al. Residential exposure to pesticide during childhood and childhood cancers: a metaanalysis. Pediatrics 2015;136:719e729.

38. Bailey HD, Fritschi L, Infante-Rivard C, et al. Parental occupational pesticide exposure and the risk of childhood leukemia in the offspring: findings from the childhood leukemia international consortium. Int $\mathrm{J}$ Cancer 2014;135:2157e2172.

39. Schüz J, Erdmann F. Environmental Exposure and Risk of Childhood Leukemia: An Overview. Arch Med Res. 2016;47(8):607-614.

40. Bailey HD, Infante-Rivard C, Metayer C, Clavel J, Lightfoot T, et al. Home pesticide exposures and risk of childhood leukemia: Findings from the childhood leukemia international consortium. Int J Cancer. 2015;137:26442663.

41. American Cancer Society. El asbesto y el riesgo de cáncer. [Internet]. USA: ACS; 2015 [Citado 12 de diciembre del 2018]. Disponible

en https://www.cancer.org/es/cancer/causas-delcancer/asbesto.html

42. Rodríguez MM. Cáncer de pulmón por hidrocarburos aromáticos policiclicos en la población de estudio CAPUA. [Tesis de posgrado]. Oviedo, España: Universidad de Oviedo; 2011 [Internet]. [Citado 27 de noviembre del 2017]. Disponible https://dialnet.unirioja.es/servlet/tesis? codigo $=98684$

43. Prazakova S, Thomas PS, Sandrini A, Yates DH. Asbestos and the lung in the 21st century: an update. Clin. Respir. J. 2014;8(1):1-10.

44. Hoy RF, Brims F. Occupational lung diseases in Australia. Med. J. Aust. 2017;207:443-448

45. Kazan-Allen L. International Ban Asbestos Secretariat. [Internet]. 2013 [citado 22 de enero de 2018]. Disponible en: http://www.ibasecretariat.org/anon-asbestos-issues-incolombia.php

46. Greenpeace. El Asbesto sigue enfermando a Colombia. Bogotá: Greenpeace; 2017. [Internet]. [Citado 27 de noviembre del 2017]. Disponible en: https://www.greenpeace.org/colombia/PageFiles/326280 /2017/6/informe-ASBESTO.pdf

47. Echegoye R, Rivera RM. Asbestosis y mesotelioma pleural maligno. Rev. Fac. Med. 2013;56(2):5-17.

48. Peters S, Glass DC, Reid A, de Klerk N, Amstrong BK, Kellie S, et al. Parental occupational exposure to engine exhausts and childhood brain tumors. Int J Cancer 2013;15(132):29752979

49. Nuyts V, Nawrot T, Nemery B, Nackaerts K. Hotspots of malignant pleural mesothelioma in Western Europe. Transl Lung Cancer Res. 2018;7(5):516-519.

50. Plato N, Martinsen JI, Kjaerheim K, Kyyronen P, Sparen P, Weiderpass E. Mesothelioma in Sweden: Dose-Response Analysis for Exposure to 29 Potential Occupational Carcinogenic Agents. Saf Health Work. 2018;9(3):290-295.

51. Kang D, Myung MS, Kim YK, Kim JE. Systematic Review of the Effects of Asbestos Exposure on the Risk of Cancer between Children and Adults. Ann Occup Environ Med. 2013;25(1):10.

52. Organización Mundial de la Salud. El tabaco es un problema que afecta a los niños en el mundo entero [Internet]. Boletín de la Organización Mundial de la Salud 2010;88:2-2. doi: 10.2471/BLT.09.069583 [citado 12 de diciembre de 2018].

Disponible https://www.who.int/bulletin/volumes/88/1/09

069583/es/

53. Observatorio del Cáncer de la AEEC. Tabaquismo y cáncer en España. Situación Actual mayo 2018. [Internet]. [citado 02 de diciembre de 2018]. Disponible en https://www.aecc.es/sites/default/files/contentfile/Informe-tabaquisimo-cancer-20182.pdf

54. Labbé A, Labbé JP. Tabaquismo pasivo en el niño. EMC Pediatría 2014;49(2):1-9

55. Peterson LA, Hecht SS. Tobacco, e-cigarettes, and child health. Curr Opin Pediatr. 2017;29(2):225-230.

56. Medina AR, Marquez CR, Torres N, Ramos L, Hernandez Y. Presencia de consumo de tabaco en un grupo de adolescentes. Gac Méd Espirit. 2015; 17(1):35-41.

57. Gibbs K, Collaco JM, McGrath-Morrow SA. Impact of Tobacco Smoke and Nicotine Exposure on Lung Development. Chest. 2016;149(2):552-561.

58. Metayer C, Zhang L, Wiemels JL, Bartley K, Schiffman J, Ma X, et al. Tobacco Smoke Exposure and the Risk of Childhood Acute Lymphoblastic and Myeloid Leukemias by Cytogenetic Subtype. Cancer Epidemiol Biomarkers Prev. 2013; 22(9):1600-11.

59. Whitehead TP, Metayer C, Wiemels JL, Singer AW, Miller MD. Childhood Leukemia and Primary Prevention. Curr Probl Pediatr Adolesc Health Care. 2016; 46(10):317-352.

60. Heck JE, Park AS, Qiu J, Cockburn M, Ritz B. Risk of leukemia in relation to exposure to ambient air toxics in pregnancy and early childhood. Int J Hyg Environ Health. 2013;217(6):662-8

61. Smith MT, Guyton KZ, Gibbons CF, Fritz JM, Portier CJ, Rusyn I, et al. Key Characteristics of Carcinogens as a Basis for Organizing Data on Mechanisms of Carcinogenesis. Environ Health Perspect. 2015; 124(6):713-21.

62. de Smith AJ, Kaur M, Gonseth S, et al. Correlates of Prenatal and Early-Life Tobacco Smoke Exposure and Frequency of Common Gene Deletions in Childhood Acute Lymphoblastic Leukemia. Cancer Res. 2017;77(7):1674-1683.

63. Williams RS, Derrick J, Phillips KJ. Cigarette sales to minors via the internet: how the story has changed in the wake of federal regulation. Tob Control. 2016;26(4):415-420.

64. Jawad M, Mclver C. Waterpipe tobacco smoking prevalence and illegal underage use in waterpipe-serving premises: a cross-sectional analysis among schoolchildren in Stoke-onTrent. Public Health 2017;146:32-38

65. Bae JM. Researches of Epigenetic Epidemiology for Infections and Radiation as Carcinogen. J Prev Med Public Health. 2018;51(4):169-172.

66. Torre LA, Bray F, Siegel RL, Ferlay J, Lortet-Tieulent J, Jemal A. Globlal cáncer statistics, 2012. CA Cancer J Clin. 2015;65(2):87-108.

67. Kamachi K, Shindo T, Miyahara M, Kitaura K, Akashi M, ShinI T, et al. Epstein - Bar virus-related diffuse large B-cell lymphoma in mogamulizumab-treated adult T-cell leukemia with incomplete $\mathrm{T}$-cell reconstitution. Int $\mathrm{J}$ Hematol 2018;109(2):221-227

68. Instituto Nacional de Cancerología de Colombia. Manual de agentes carcinógenos de los grupos 1 y 2 a de la IARC, de interés ocupacional para Colombia. [Internet] [Citado 20 de marzo de 2018]. Disponible en: http://www.cinu.mx/comunicados/ManualAgentes.pdf

69. Lorenzo I, Fernandez de Larrea N, Michel A, Romero B, Lope V, Bessa X, et al. Helicobacter pylori seroprevalence in Spain: Influence of adult and childhood sociodemographic factors. Eur J Cancer Prev. 2019;28(4):294-303. 